\title{
Saber Tradicional y Prácticas en Odontología. Su Asocia- ción con la Caries Dental de una Población Escolar
}

\author{
Traditional Knowledge and Practices in Dental Health. \\ Their Association to Caries in a School Population
}

María del Pilar Adriano Anaya*; Tomás Caudillo Joya* \& Pilar Alejandra Caudillo Adriano**

ADRIANO, A. M. P.; CAUDILLO, J. T. \& CAUDILLO, A. P. A. Saber tradicional y prácticas en odontología. Su asociación con la caries dental de una población escolar. Int. J. Odontostomat., 10(3):455-462, 2016.

RESUMEN: El objetivo de este trabajo fue identificar el saber tradicional en Odontología de padres de familia en una población escolar de la delegación Iztapalapa en la Ciudad de México, así como las prácticas y tipo de quehacer para resolver sus problemas orales y su asociación con la caries dental. Se llevó a cabo un estudio transversal, descriptivo y observacional realizado en 1474 padres de familia con sus respectivos hijos inscritos en diez escuelas primarias públicas, previa información del estudio y su autorización para su participación. Los resultados se capturaron en el programa de cómputo SPSS versión 15.0. Cuando se preguntó a los padres que es la caries dental, contestaron 36 opciones diferentes, el $25 \%$ contestaron es cuando se pican o se hacen hoyos en los dientes. El $68 \%$ de los padres explicaron que los motivos por lo que se pican los dientes es por mala higiene. El 60 \% de ésta población únicamente asiste al Dentista por necesidades sentidas. Identificar el saber tradicional en Odontología de padres de familia en una población escolar de la delegación Iztapalapa en la Ciudad de México, así como las prácticas y tipo de quehacer para resolver sus problemas bucales, nos permite conocer el conocimiento que tiene la población con respecto a su salud bucal y retroalimentar el conocimiento científico, donde ambos actores sociales se transforman.

PALABRAS CLAVE: saber tradicional, caries dental, escolares, prevención.

\section{INTRODUCCIÓN}

La caries dental es un problema específico del Proceso Salud Enfermedad Estomatológico que se encuentra multideterminado por factores económicos, políticos, sociales, biológicos y culturales entre otros, y que se manifiesta a nivel individual por la desmineralización del esmalte de los dientes por mecanismos de acción bacteriana, formando así a la caries dental (Adriano, 2013; Adriano Anaya et al., 2014).

La OMS ha declarado que se estima que cinco mil millones de personas en el planeta han sufrido caries dental. Los efectos de las enfermedades bucodentales en términos de dolor, sufrimiento, deterioro funcional y disminución de la calidad de vida son considerables y costosos. Aproximadamente, el tratamiento representa entre el $5 \%$ y el $10 \%$ del gasto sanitario de los países industrializados, y está por en- cima de los recursos de muchos países en desarrollo (Adriano Anaya et al., 2003; Adriano Anaya \& Caudillo Joya, 2015).

Sin embargo es necesario el reconocer que 43,4 millones de mexicanos no tienen acceso a ninguna institución o programa de Salud Pública o privada (Agudelo \& Martínez, 2005), lo que ha llevado a que cada vez la población acuda a la práctica tradicional para resolver sus problemas bucales, y en particular a la caries dental y de ahí la importancia de conocer y reconocer lo que la población piensa sobre este problema, las medidas de prevención para su control y las instituciones a las que acuden para su solución.

Como Odontólogos, es un desafío el reconocer este conocimiento y entender que las comunidades

\footnotetext{
* Facultad de Estudios Superiores Zaragoza UNAM, México.

* Universidad Autónoma Metropolitana, México.
} 
son igualmente productoras de saberes tradicionales bucales, y que no existe únicamente el conocimiento científico, reconocido por las instituciones formadoras de sujetos humanos en Odontología, sino que debemos articularlos como un proceso de comunicación en que ambos se reconozcan, se respeten y se transformen mutuamente estamos hablando en consecuencia de un diálogo de saberes.

Delgado (Arango Arroyave \& Iságama, 2012) Establece que el diálogo de saberes es necesario para reorganizar los conocimientos, producirlos mediante el cambio de perspectivas, comportamientos y modos de pensar y colaborar.

El diálogo de saberes en Ciencias de la Salud y específicamente en Odontología, lo vamos a entender como un proceso complejo e intercultural donde se fortalecen y complementan los conocimientos científicos con las respuestas generadas en homeopatía, herbolaria, acupuntura, y el tradicional que se hereda de padres a hijos, que han sido marginados por el saber hegemónico científico, sin embargo son saberes que se sustentan en una visión del mundo y en particular en salud bucal es decir son posturas culturales para poder resolver los problemas del proceso salud-enfermedad bucal con respeto a la cultura, valores, símbolos, tradiciones, en donde exista un empoderamiento de los actores sociales sobre su comprensión (Bastidas Acevedo et al., 2009; Caudillo et al., 2013). El Odontólogo en el quehacer clínico y en las comunidades no debe intervenir imponiendo su conocimiento, sino al contrario propiciar el diálogo con la población en estudio, escuchar, respetar y reconocer a la población, donde interprete y exprese sus significados sobre los problemas bucales y presente la forma como resuelven sus problemas, ya que ellos son poseedores de su propio conocimiento. En consecuencia se rebasa la visión sujeto-objeto por la de sujeto-sujeto.

Es necesario buscar las estrategias que posibiliten un acercamiento de tipo intercultural entre la institucionalidad y las comunidades, que debe encararse como un reto que impone nuevas miradas y reconocimiento del saber tradicional, en diálogo con el conocimiento formal o científico (Delgado Díaz, 2012).

El diálogo de saberes sobre el proceso saludenfermedad bucal, no tiene como fin lograr que el otro piense $o$ actúe de una manera determinada, pues se basa en el respeto y en el fortalecimiento de la autonomía. Requiere en sí, el respeto de las diferencias bajo un consenso básico: la posibilidad de expresar libre- mente y sin coacciones las posiciones, intereses, inquietudes y necesidades. implica reconocer al otro como individuo, como sujeto con capacidades para construir una visión propia y actuar con criterio (Organización Mundial de la Salud, 2007), tomando en cuenta las circunstancias en que las personas nacen, crecen, viven, trabajan y envejecen, incluido el sistema de salud, esto es sus determinantes sociales (Heredia, 2006; Norma Oficial Mexicana, 2012).

Dentro de los estudios que tratan de ampliar el contexto explicativo de la salud-enfermedad bucal, en el año 2005, se realizó una investigación, enfocada en los conocimientos, actitudes, prácticas y algunas expectativas frente a la salud bucal, medios de comunicación, y mercadeo social en los niños de 7 a 12 años de la escuela Rafael Uribe de la ciudad de Medellín, realizando asociaciones entre la caries dental y su etiología. Esta población respondió que la noche es el tiempo más importante para el lavado de los dientes. Cada uno tiene su propia percepción del tiempo para cambiar el cepillo, pues lo que para unos niños, 3 meses puede ser viejo, para otros pueden pasar 2 años sin considerar que su cepillo necesita cambio. Sería interesante que consideraran otras características como el hecho de que su cepillo esté deshilachado para saber que ya no está en condiciones de realizar bien su función (Organización Panamericana de la Salud, 2004).

Otro estudio descriptivo transversal en la población de 15-18 años con el objetivo de determinar el nivel de conocimiento sobre salud buco dental, en la población de Barrio Adentro Peribeca. Municipio Independencia. San Cristóbal. Estado Táchira Venezuela, se obtuvo un nivel de conocimiento malo sobre salud buco dental en la población objeto de estudio. Se recomienda, incrementar las acciones de prevención y promoción de salud encaminadas a elevar la educación sanitaria desde edades tempranas de la vida (Rocabado, 2005).

Un estudio realizado en México (Caudillo et al.) sobre los saberes científicos y tradicionales, en alumnos que cursan la Carrera de Cirujano Dentista en la Facultad de Estudios Superiores Zaragoza UNAM, el $42,6 \%$ de los alumnos cepilla sus dientes dos veces al día, por la mañana y noche. Casi la mitad de los alumnos cuenta con buenos hábitos para el control de los problemas bucales, por lo que se debe trabajar de una manera sistemática en el $50 \%$ restante de los alumnos, para generar una cultura de la salud bucal. Para el cepillado de los dientes utilizan la pasta dental. 


\section{MATERIAL Y MÉTODO}

Estudio epidemiológico, de tipo cuali-cuantitativo sobre el saber tradicional que tienen los padres de familia o tutores de los escolares de seis a doce años de diez escuelas primarias ubicadas en la delegación Iztapalapa localizada al oriente de la Ciudad de Méxi$\mathrm{co}$, y que se encuentran dentro del programa de salud bucal que la carrera de Cirujano dentista de la Facultad de Estudios Superiores Zaragoza está implementando en convenio con la Secretaría de Educación Pública de esta Delegación.

Para poder realizar este estudio se reunió a los padres de familia donde se platicó con ellos sobre el objetivo del mismo y que los resultados servirían para desarrollar un programa de protección específica y otro de promoción de la salud bucal en sus hijos, con el propósito de disminuir la caries dental en un $50 \%$ y generar en ellos una cultura de la salud bucal, para lo cual se elaboró un cuestionario con nueve preguntas dos de ellas abiertas y siete cerradas, sobre la causa (conocimiento), prevención y servicio demandado para resolver sus problemas odontológicos. La muestra por conveniencia se conformó con 1474 padres o tutores que dieron su consentimiento por escrito para participar en este estudio y resolvieron el cuestionario.

Posteriormente se llevó a cabo la revisión bucal de sus hijos. El indicador epidemiológico utilizado fue el $C P O D(C=$ cariados, $\mathrm{P}=$ perdidos, $\mathrm{O}=$ obturados $\mathrm{D}=$ diente), siguiendo las recomendaciones de la Organización Mundial de la Salud (OMS) (Petersen, 2003) debido a que es mundialmente confiable, estandarizado y permite la comparación con otros estudios (World Health Organization, 2013). La exploración bucal la realizaron cinco pasantes de la carrera de Cirujano Dentista y dos profesores responsables de este estudio, que fueron entrenados y calibrados en el conocimiento y aplicación de los códigos y criterios del indicador CPOD. Con el propósito de que la concordancia no se atribuyera al azar, se aplicó el coeficiente de KAPPA Intra e interexaminadores que permite medir las variaciones de las observaciones realizadas por los revisores (K=0,84; IC $95 \% 0,43-$ $0,81)$. La revisión bucal, se llevó a cabo en aulas de clase, adaptándonos a las condiciones de cada una de las primarias, (sillas, mesas, pupitres, bancas), los recursos utilizados fueron: espejos dentales, sonda WHO, pinzas de curación y luz natural, aplicando las barreras de protección planteadas por la Norma Oficial Mexicana para el control y manejo de infecciones bucales, NOM-087-ECOL-SSA1-2002 (Norma Oficial Mexicana).

Con respecto a las preguntas abiertas, las respuestas se fueron concentrando de acuerdo a conceptos integradores y conservando aquellas cuyas respuestas fueron únicas. Con relación a las cerradas únicamente se contabilizaron. La información de todas las variables del estudio se capturaron en el Software estadístico SPSS versión 17.0. Se obtuvo porcentajes totales de las preguntas y promedios del indicador que nos midió la caries dental. Se obtuvieron pruebas de significancia estadística.

\section{RESULTADOS}

La primera pregunta realizada a los padres de familia fue: Explique brevemente que es la caries dental. Las respuestas se concentraron en 36 opciones diferentes.

Respondieron 368 es cuando se pican o se hacen hoyos en los dientes. 275 padres respondieron que era una enfermedad producida por bacterias, microbios, gusanos o bichos. 224 es enfermedad o infección en los dientes. 113 contestaron daño producido por mal cuidado o mala higiene. 40 enfermedad producida por dulces. Del resto, sus respuestas fueron diversas como pudrición, destrucción, lesión, masa, mancha de los dientes. Con respecto a los promedios de caries dental fueron muy similares no encontrandose diferencias estadísticas $p=0.426$ (Tabla I).

El $68 \%$ de los padres explicaron que los motivos por lo que se pican los dientes es por mala higiene, el $24 \%$ por consumir alimentos con azúcar o mala alimentación. El resto por diversas causas. (Tabla II). Al asociar los saberes de los padres con los promedios de caries dental de los hijos se encontró asociación entre ambas variables.

El $60 \%$ de ésta población únicamente asiste al Dentista por necesidades sentidas, esto es por dolor o bien nuca ha asistido, el $60 \%$ restante respondió asistir de una a dos veces al año. No se encontró asociación entre las opciones que respondieron con los promedios de caries dental. $p=0,810$. (Tabla III).

Los motivos por los que esta población visita al dentista es por dolor, que no les permite realizar sus 
Tabla I Explique brevemente que es la caries dental.

\begin{tabular}{|c|c|c|c|c|c|}
\hline Frec. & Opciones & No. & $\%$ & CPOD & DS \\
\hline 1 & Es el daño producido por mala higiene & 78 & 5,3 & 3,0 & 2,3 \\
\hline 2 & Es el exceso de azúcar que comemos & 21 & 1,4 & 3,7 & 2,4 \\
\hline 3 & Es una mancha que pica los dientes & 83 & 5,6 & 2,9 & 2,1 \\
\hline 4 & Es la pudrición de los dientes & 16 & 1,1 & 2,4 & 1,4 \\
\hline 5 & Es la enfermedad en los dientes & 94 & 6,4 & 3,2 & 1,8 \\
\hline 6 & Es cuando se pican los dientes & 291 & 19,7 & 3,0 & 2,1 \\
\hline 7 & Es la comida acumulada en los dientes & 23 & 1,6 & 2,8 & 2,4 \\
\hline 8 & Es la enfermedad producida por bacterias & 140 & 9,5 & 2,9 & 2,3 \\
\hline 9 & Es cuando se pican los dientes y se inflaman las encías & 18 & 1,2 & 2,7 & 1,6 \\
\hline 10 & Es destrucción del tejido dental & 57 & 3,9 & 3,0 & 1,9 \\
\hline 11 & Cuando se pega una cosa negra en los dientes & 16 & 1,1 & 1,9 & 1,8 \\
\hline 12 & Son bacterias que viven en los dientes y salen por comer dulces & 72 & 4,9 & 3,0 & 1,6 \\
\hline 13 & Cuando salen puntos negros en los dientes & 81 & 5,5 & 2,9 & 1,9 \\
\hline 14 & Es la masa en los dientes & 6 & 0,4 & 2,7 & 1,7 \\
\hline 15 & Es enfermedad por masticar los dulces & 9 & 0,6 & 2,2 & 1,8 \\
\hline 16 & No sabe & 92 & 6,2 & 2,6 & 2,2 \\
\hline 17 & Cuando los dientes se dañan por falta de cuidado & 12 & 0,8 & 3,3 & 3,2 \\
\hline 18 & Es cuando se hace sarro en los dientes & 36 & 2,4 & 2,4 & 1,4 \\
\hline 19 & Es una mancha en los dientes & 33 & 2,2 & 2,9 & 2,3 \\
\hline 20 & Es una infección en los dientes & 60 & 4,1 & 3,0 & 2,4 \\
\hline 21 & Son problemas de la dentadura & 9 & 0,6 & 2,8 & 2,5 \\
\hline 22 & Es un microbio que devora el diente & 28 & 1,9 & 2,9 & 2,0 \\
\hline 23 & Es la enfermedad por exceso de dulces y chocolate & 10 & 0,7 & 3,4 & 2,1 \\
\hline 24 & Es el trastorno común después del resfriado & 7 & 0,5 & 2,6 & 1,3 \\
\hline 25 & Es una lesión en los dientes & 4 & 0,3 & 2,5 & 1,7 \\
\hline 26 & Es la placa bacteriana de los dientes & 11 & 0,7 & 1,9 & 1,6 \\
\hline 27 & Es todo lo que comemos y se va acumulando & 11 & 0,7 & 3,4 & 2,2 \\
\hline 28 & Es el hoyo en los dientes & 55 & 3,7 & 2,7 & 1,8 \\
\hline 29 & Deterioro del diente & 14 & 0,9 & 3,1 & 2,4 \\
\hline 30 & Ácidos de los alimentos que dañan al diente & 13 & 0,9 & 4,0 & 3,5 \\
\hline 31 & Gusano que se come los dientes & 10 & 0,7 & 3,0 & 1,9 \\
\hline 32 & Bichos que se comen los dientes & 25 & 1,7 & 4,1 & 2,2 \\
\hline 33 & Puntos amarillos en los dientes & 9 & 0,6 & 3,6 & 2,3 \\
\hline 34 & Mugre que daña al diente & 16 & 1,1 & 2,4 & 1,3 \\
\hline 35 & Enfermedad multifactorial & 10 & 0,7 & 3,9 & 1,6 \\
\hline \multirow[t]{2}{*}{36} & Es una bola negra que sale en los dientes & 4 & 0,3 & 2,5 & 1,2 \\
\hline & Total & 1474 & 100,0 & & $\begin{array}{c}2,1 \\
p=0,426\end{array}$ \\
\hline
\end{tabular}

actividades normales y sus funciones de la cavidad oral, un hecho a resaltar es la poca importancia que esta población le da a su salud oral donde sólo el 11,1 $\%$ asiste al dentista para recibir acciones de prevención. Al asociar las respuestas con los promedios de caries dental no se encontró asociación $p=0,377$. (Tabla IV).

En esta población hay padres de familia que no cuentan con cepillo dental $(0,5 \%)$, o bien existe uno para toda la familia. $(0,7 \%)$. Esto puede atribuirse a los limitados recursos económicos con los que cuenta esta población que no les permite comprar cepillos den- tales y a la pobre cultura de su salud bucal, al asociar esta pregunta con los promedios de caries dental no se encontró significancia estadística. (Tabla V).

La población tiene el conocimiento de que los cepillos dentales hay que cambiarlos cada tres meses, porque esa es la información que recibimos de la televisión, sin embargo una cosa es el conocimiento, el deseo y otra la realidad ya que al asociar con los promedios de caries dental si hubiera correspondencia entre el cambio de cepillo y el cepillado en estos escolares el promedio de caries dental debía ser el más bajo y no el más alto como se puede apreciar. (Tabla VI). 
Tabla II. Pregunta 2. Explique brevemente por qué motivos se pican los dientes

\begin{tabular}{lcccc}
\hline Por mala higiene & 636 & 2,7 & 1,7 & 38,1 \\
Por exceso de azúcar & 149 & 2,9 & 2,0 & 9,9 \\
Por no cepillarse los dientes & 315 & 3,2 & 2,3 & 23,7 \\
no sabe & 17 & 4,2 & 2,2 & 1,6 \\
Por comer mucho dulce, golosinas, chatarra y falta de higiene & 177 & 3,2 & 2,0 & 12,6 \\
Por mal cepillado & 52 & 3,3 & 2,5 & 3,9 \\
Por falta de calcio & 24 & 3,1 & 2,7 & 1,7 \\
Por consumir muchos alimentos con sal y azúcar & 18 & 2,9 & 1,9 & 1,2 \\
Por la herencia & 18 & 3,1 & 2,7 & 1,3 \\
Por mala alimentación & 11 & 2,4 & 3,0 & 0,6 \\
Por no tener cuidados en la boca & 11 & 5,9 & 3,7 & 1,5 \\
Por ignorancia & 6 & 3,5 & 1,7 & 0,5 \\
Por no ir al dentista & 40 & 3,6 & 2,5 & 3,3 \\
Total & 1474 & & 2,123 & 100,0 \\
& & & & $\mathrm{p}=0,000$ \\
\hline
\end{tabular}

Tabla III. Pregunta 3. ¿Cada cuanto tiempo visita al Dentista?

\begin{tabular}{lcccc}
\hline Opciones & No. & $\%$ & CPOD & DS \\
\hline Nunca & 208 & 14,1 & 2,8 & 2,0 \\
Una vez al año & 559 & 37,9 & 3,0 & 2,2 \\
Sólo cuando tengo dolor & 686 & 46,5 & 3,0 & 2,0 \\
Dos veces al año & 7 & 0,5 & 2,6 & 1,3 \\
Cada seis meses & 14 & 0,9 & 2,6 & 1,9 \\
Total & 1474 & 100 & 2,94 & 2,1 \\
& & & & $p=0,810$ \\
\hline
\end{tabular}

Tabla V. Pregunta 5. ¿En su familia todos tienen cepillo dental?.

\begin{tabular}{lcccc}
\hline Opciones & CPOD & $\mathrm{N}$ & $\mathrm{SD}$ & $\%$ \\
\hline Si & 2,9 & 1450 & 2,1 & 98,6 \\
Hay un cepillo dental para & 2,4 & 15 & 2,0 & 0,7 \\
toda la familia & 3,1 & 2 & 1,4 & 0,1 \\
Solo papá tiene cepillo & 3,2 & 7 & 2,1 & 0,5 \\
Nadie tiene & 2,9 & 1474 & 2,1 & 100,0 \\
Total & & & \multicolumn{3}{r}{$\mathrm{P}=0,534$} \\
\end{tabular}

Tabla IV. Pregunta 4. ¿Porqué motivos visita al Dentista?

\begin{tabular}{lcccc} 
Opciones & No. & $\%$ & CPOD & DS \\
\hline Dolor & 681 & 46,7 & 3,0 & 2,095 \\
Por tratamiento & 203 & 14,3 & 3,1 & 2,117 \\
Por prevención & 165 & 11,7 & 3,1 & 2,034 \\
Revisión & 248 & 16,1 & 2,8 & 2,301 \\
No lo visitó & 177 & 11,1 & 2,7 & 2,057 \\
Total & 1474 & 100 & & 2,123 \\
& & & & $\mathrm{P}=0,377$
\end{tabular}

Tabla VI. Pregunta 6. ¿Cada cuanto tiempo cambia su cepillo dental?.

\begin{tabular}{lcccc}
\hline Opciones & CPOD & No. & DS & $\%$ \\
\hline Nunca & 2,1 & 31 & 2,055 & 1,5 \\
Cada seis meses & 1,6 & 303 & 1,732 & 11,0 \\
Una vez al año & 2,3 & 51 & 1,426 & 2,7 \\
Cuando ya no sirve & 2,4 & 401 & 1,484 & 22,5 \\
Cada tres meses & 3,9 & 688 & 2,171 & 62,3 \\
Total & 2,94 & 1474 & 2,123 & 100,0 \\
& & & & $P=0,000$ \\
\hline
\end{tabular}


El 30,4 \% de esta población resuelve sus problemas bucales en los Centros de Salud, que forman parte de la Secretaría de Salud y que el servicio se proporciona a las personas que compran el seguro popular. Podemos observar la poca cobertura que tienen las clínicas de la Universidad Nacional Autónoma de México, donde sólo el 3,2 \% de esta población solicita este servicio. (Tabla VII).

Más de la mitad $(56,4 \%)$ cepilla sus dientes dos veces al día, encontrando que el $4,6 \%$ cepilla sus dientes de vez en cuando. Podemos observar que no hay correspondencia entre los que dicen cepillar sus dientes tres veces al día y su promedio de caries dental siendo el más elevado con 3,2 dientes con historia de esta enfermedad. Se encontró significancia estadística entre la frecuencia de cepillado y los promedios de caries dental $(p=0,001)$. (Tabla VIII).

El 2,7\% de esta población cepilla sus dientes con materiales como es el bicarbonato, la ceniza del fogón o bien la tortilla quemada, consideramos que se da esta situación por la falta de recursos económicos. No se encontró asociación con los promedios de la caries dental $(p=0,690)$. (Tabla IX).

Tabla VII Pregunta 7. Cuando acude al Dentista ¿a qué institución lo hace?.

\begin{tabular}{lcccc}
\hline Opciones & CPOD & N0. & DS & $\%$ \\
\hline Centro de salud & 3,0 & 439 & 2,2 & 30,4 \\
IMSS & 3,1 & 132 & 2,0 & 9,4 \\
ISSSTE & 3,0 & 52 & 2,3 & 3,5 \\
Dispensario & 3,2 & 67 & 2,4 & 5,0 \\
Curandero & 2,7 & 11 & 3,1 & 0,7 \\
Privado & 3,0 & 413 & 2,0 & 27,7 \\
Clínicas UNAM & 3,5 & 40 & 2,1 & 3,2 \\
Otro & 3,1 & 46 & 2,0 & 3,3 \\
Ninguno & 2,7 & 274 & 1,9 & 16,9 \\
Total & 2,94 & 1474 & 2,1 & 100 \\
& & & & $\mathrm{P}=0,311$ \\
\hline
\end{tabular}

ISSSTE= Instituto de Seguridad y Servicios Social para los Trabajadores del Estado

IMSS = Instituto Mexicano del Seguro Social

UNAM= Universidad Nacional Autónoma de México
Tabla VIII El cepillado de sus dientes lo realiza usted

\begin{tabular}{lcccc}
\hline Opciones & CPOD & No. & DS & $\%$ \\
\hline Sólo en la noche & 2,3 & 76 & 2,0 & 4,1 \\
Mañana y noche & 3,0 & 825 & 2,1 & 56,4 \\
Mañana & 2,8 & 124 & 2,1 & 8,0 \\
Tres veces al día & 3,2 & 365 & 2,1 & 27,0 \\
De vez en cuando & 2,4 & 84 & 1,7 & 4,6 \\
Total & & 1474 & 2,1 & 100 \\
& & & & $P=0,001$
\end{tabular}

Tabla IX. Pregunta 9. ¿Qué utiliza para el cepillado de sus dientes?

\begin{tabular}{lcccc}
\hline Opciones & CPOD & No. & DS & $\%$ \\
\hline Pasta & 2,94 & 1432 & 2,143 & 97,3 \\
Bicarbonato & 3,00 & 13 & 1,225 & 0,9 \\
Ceniza del fogón & 3,22 & 9 &, 972 & 0,7 \\
Tortilla quemada & 2,40 & 20 & 1,429 & 1,1 \\
Total & 2,94 & 1474 & 2,123 & 100 \\
& & & \multicolumn{3}{c}{$\mathrm{P}=0,690$} \\
\hline
\end{tabular}

\section{DISCUSIÓN}

Con el propósito de que el proceso salud-enfermedad del sistema estomatognático se exprese como dinámicas históricas, donde el sujeto interactúa con el contexto a partir de retroacciones provocando una relación sujeto-sujeto, la salud ya no es entendida como un estado; sino como un proceso en construcción permanente en el que se reconoce lo cultural y lo social como manifestaciones simbólicas y materiales que comprometen todas las relaciones del hombre y por lo tanto de su vida misma.

Cuando se consideran las instituciones que intervienen para responder a las problemáticas de la salud-enfermedad se mencionan a las asistenciales, institucionales y privadas marginando a las tradicionales, a las que han surgido como producto de la experiencia de la propia sociedad producto de su propia historia, consideramos que es indispensable el incorporarlas debido a estas resuelven en alguna medida algunos de sus problemas. Es importante incorporar a la cultura y a la subjetividad como elementos que intervienen en la comprensión de los problemas de la salud-enfermedad. 
Es en este contexto en que habrá que darle sentido a las respuestas que proporcionaron los padres de familia o tutores de los escolares que intervinieron en el estudio.

Con respecto a los conocimientos que tienen sobre la caries dental, se puede hacer notar la diversidad de visiones que tienen sobre esta, sin lugar a dudas es importante el considerar el punto de vista que tienen, ya que es el reflejo de la realidad en el que ellos se desarrollan. Tomando en consideración esa vista de punto que los padres tienen sobre la causa por la que se pican los dientes se ve reflejada también la información con la que cuentan para poder explicar el origen de esta. Un reflejo de como la cultura interviene sobre el problema de la caries dental, es la actitud que toman al momento en el que hay que resolver cuando se presenta esta, solo acuden al dentista cuando existe una necesidad sentida, es decir cuando existe un problema de dolor, no asisten al dentista para revisión o para evitar la aparición de esta, es decir, que la salud bucal no es importante para ellos, lo es, cuando se presenta algún problema que es necesario resolver, y cuando se presenta este problema prevalece la participación del profesional en la solución de este, pero en esta población resuelve en un porcentaje importante el que ejerce una práctica tradicional como es el curandero.

La información que refleja este estudio se aprecia la multidimensionalidad en el origen de los problemas de la salud-enfermedad bucal en particular de la caries dental, los que habrá que tomar en cuenta cuando se propongan acciones de intervención para poder modificarla o transformarla.

\section{CONCLUSIÓN}

Articular el saber tradicional en Odontología de padres de familia en una población escolar de la delegación Iztapalapa en la Ciudad de México, así como sus prácticas y tipo de quehacer que utilizan para resolver sus problemas bucales, nos permite identificar el conocimiento que tiene la población con respecto a su salud bucal y retroalimentar el conocimiento científico, donde ambos actores sociales se transforman y con ello ampliar los marcos explicativos de la saludenfermedad bucal y en consecuencia generar respuestas sociales a partir de programas de prevención, promoción y rehabilitación acorde a las necesidades bucales de cada grupo poblacional.
ADRIANO, A. M. P.; CAUDILLO, J. T. \& CAUDILLO, A. P. A. Traditional knowledge and practices in dental health. Their association to caries in a school population. Int. J. Odontostomat., 10(3):455-462, 2016.

ABSTRACT: The objective of this work was to identify the traditional knowledge of dental health of parents, in a school population of the Iztapalapa delegation in Mexico City, as well as their practices and how they handle solving oral health problems, as well as their association with dental caries. A cross-sectional descriptive and observational study was carried out in 1474 parents with their respective children enrolled in ten public elementary schools, after receiving information about the study and authorization to participate. The results were captured in the SPSS software version 15.0. When parents were asked what tooth decay is, they answered from 36 different options; $25 \%$ answered when teeth are decayed or punctured. $68 \%$ of the parents explained that the reasons teeth are decayed is poor hygiene. $60 \%$ of this population only attends the Dentist when the need arises. To identify the traditional knowledge of parents with respect to oral health in a school population of the Iztapalapa delegation in Mexico City, as well as the practices and management to solve their oral health problems, allows us access to information of the knowledge this population has with respect to their oral health and provides scientific feedback, where both social participants are transformed.

KEY WORDS: traditional knowledge, dental caries, school population, prevention.

\section{REFERENCIAS BIBLIOGRÁFICAS}

Adriano, M. P. Pensamiento Complejo en Odontología. Tesis Doctoral. Ciudad de México, Multiversidad Mundo Real Edgar Morin, 2013. pp.35.

Adriano Anaya, M. P.; Caudillo Joya, T.; Malinowski, N. \& Caudillo Adriano, P. A. Scientific and traditional knowledge in odontology. Open J. Stomatol., 4(7):358-71, 2014.

Adriano Anaya, M. P. \& Caudillo Joya, T. Complejidad del Proceso Salud Enfermedad Estomatológico. Ciudad de México, Facultad de Estudios Superiores Zaragoza UNAM, 2015. pp.74-5.

Adriano Anaya, M. P.; Caudillo Joya, T. \& Gómez, C. A. Epidemiologia Estomatológica. Ciudad de México, Facultad de Estudios Superiores Zaragoza UNAM, 2003. pp.8-14.

Agudelo, S. A. A. \& Martínez, R. L. I. Conocimientos, actitudes y prácticas en salud bucal, medios de comunicación y mercadeo social en escolares de 7 a 12 años de una institución de Medellín. Rev. Fac. Odontol. Univ. Antioq., 16(1-2):81-94, 2005. 
ADRIANO, A. M. P.; CAUDILLO, J. T. \& CAUDILLO, A. P. A. Saber tradicional y prácticas en odontología. Su asociación con la caries dental de una población escolar Int. J. Odontostomat., 10(3):455-462, 2016.

Arango Arroyave, J. U. \& Iságama, M. E. Flora etnoodontológica de las comunidades indígenas embera del Atrato Medio antioqueño. Rev. Fac. Odontol. Univ. Antioq., 23(2):321-33, 2012.

Bastidas Acevedo, M.; Pérez Becerra, F. N.; Torres Ospina, J. N.; Escobar Paucar, G.; Arango Córdoba, A. \& Peñaranda Correa, F. El diálogo de saberes como posición humana frente al otro: referente ontológico y pedagógico en la educación para la salud. Investig. Educ. Enferm., 27(1):104-111, 2009.

Caudillo, T.; Adriano, P. \& Gurrola, B. Formando Promotores de la Salud Bucal. Ciudad de México, Facultad de Estudios Superiores Zaragoza UNAM, 2013. pp.8-9.

Delgado Díaz, C. J. Conocimiento, conocimientos, diálogo de saberes. Ruth, (10):159-80, 2012.

Organización Mundial de la Salud (OMS). Determinantes Sociales de la Salud. Ginebra, Comisión sobre Determinantes Sociales de la Salud (CDSS) Evidencia e Información para Políticas, OMS, 2007. Disponible en: http:/ /www.who.int/social_determinants/es/

Heredia, J. M. Nivel de Conocimiento sobre Salud Bucal en la Población de 15-18 años. Barrio Adentro Peribeca. Monografía en Internet. (Monografía en Internet). Táchira, Revista Ciencias.com, 2006. Disponible en: http://www.ilustrados.com/tema/10589/Nivel-conocimiento-sobre-salud-bucal-poblacion.html

Norma Oficial Mexicana NOM-087-ECOL-SSA1-2002, protección ambiental-residuos peligrosos biológico-infecciosos-clasificación y especificaciones de manejo. Ciudad de México, MAYPA, 2012.

Organización Panamericana de la Salud (OPS). La OMS Publica un Nuevo Informe sobre el Problema Mundial de las Enfermedades Bucodentales. La Paz, Organización Panamericana de la Salud, 2004. Disponible en: h t t p : / / w w w. p a ho.o r g / b o l / index.php? option =com_content\&view = article \&id $=278 \&$ catid $=6 \overline{6} 7 \% 3$ Anotas $-d e-$ prensa\&ltemid=1

Rocabado, F. Determinantes Sociales de la Salud en Perú. Cuadernos de promoción de la salud No. 17. Ministerio de Salud. Lima Edic. María Edith Baca. 25-77, 2005.

Petersen, P. E. The World Oral Health Report 2003: continuous improvement of oral health in the 21 st century-the approach of the WHO Global Oral Health Programme. Community Dent. Oral Epidemiol., 31 Suppl. 1:3-23, 2003.

World Health Organization (WHO). Oral Health Surveys: Basic Methods. 5th ed. Ginebra, World Health Organization, 2013.
Dirección paraorrespondencia:

Maria del Pilar Adriano Anaya

Facultad de Estudios Superiores Zaragoza UNAM

Universidad de Autónoma de México

MÉXICO

Email: adriano124@hotmail.com

Recibido : 28-08-2016

Aceptado: 22-09-2016 